\title{
Prediction of Neutron Yield of IR-IECF Facility in High Voltages Using Artificial Neural Network
}

\author{
A. Sadighzadeh, ${ }^{1}$ A. Salehizadeh, ${ }^{1,2}$ M. Mohammadzadeh, ${ }^{3}$ F. Shama, ${ }^{4}$ S. Setayeshi, ${ }^{5}$ \\ S. A. H. Feghhi, ${ }^{3}$ S. M. Sadati, ${ }^{3}$ M. Rezaei, ${ }^{3}$ E. Haji Ebrahimi, ${ }^{5}$ and G. H. Roshani ${ }^{1,3}$ \\ ${ }^{1}$ Plasma and Fusion Research School, Nuclear Science and Technology Research Institute, Tehran, Iran \\ ${ }^{2}$ Faculty of New Science and Technology, University of Isfahan, Isfahan, Iran \\ ${ }^{3}$ Department of Radiation Application, Shahid Beheshti University, GC, Tehran, Iran \\ ${ }^{4}$ Young Researchers and Elite Club, Islamic Azad University, Kermanshah Branch, Kermanshah, Iran \\ ${ }^{5}$ Department of Energy Engineering and Physics, Amirkabir University of Technology, Tehran, Iran
}

Correspondence should be addressed to G. H. Roshani; hosseinroshani@yahoo.com

Received 18 July 2014; Revised 2 November 2014; Accepted 2 November 2014; Published 16 December 2014

Academic Editor: Jyh-Hong Chou

Copyright (C) 2014 A. Sadighzadeh et al. This is an open access article distributed under the Creative Commons Attribution License, which permits unrestricted use, distribution, and reproduction in any medium, provided the original work is properly cited.

\begin{abstract}
Artificial neural network (ANN) is applied to predict the number of produced neutrons from IR-IECF device in wide discharge current and voltage ranges. Experimentally, discharge current from 20 to $100 \mathrm{~mA}$ had been tuned by deuterium gas pressure and cathode voltage had been changed from -20 to $-82 \mathrm{kV}$ (maximum voltage of the used supply). The maximum neutron production rate $(\mathrm{NPR})$ of $1.46 \times 10^{7} \mathrm{n} / \mathrm{s}$ had occurred when the voltage was $-82 \mathrm{kV}$ and the discharge current was $48 \mathrm{~mA}$. The back-propagation algorithm is used for training of the proposed multilayer perceptron (MLP) neural network structure. The obtained results show that the proposed ANN model has achieved good agreement with the experimental data. Results show that NPR of $1.855 \times 10^{8} \mathrm{n} / \mathrm{s}$ can be achieved in voltage and current of $125 \mathrm{kV}$ and $45 \mathrm{~mA}$, respectively. This prediction shows $52 \%$ increment in maximum voltage of power supply. Also, the optimum discharge current can increase $1270 \%$ NPR.
\end{abstract}

\section{Introduction}

Inertial electrostatic confinement fusion (IECF) device is a compact and simple structure for nuclear fusion researches by electrical discharge, which can operate in the pulsed or continuous mode. It consists of two concentric (or coaxial) electrodes in which usually the central one (cathode) is negatively high-voltage-biased and the outward electrode (anode) is grounded. In this configuration, strong electric fields between electrodes lead to iodinating the filling gas and then accelerate the created ions toward the center, where the electrons are placed in the opposite direction. As a result, rather hot and dense plasma is formed in the center of cathode. In this situation, the continuous nuclear fusion reactions occur, which are the results of the beam-target and beam-beam interactions depending on the working conditions, although the beam-beam interactions will be negligible compared to beam-target reactions in the plasma conditions in this study.
Therefore IECF is considered as a source of hot and dense plasma, highly energetic ions, and fast neutrons (when using deuterium or mixture of deuterium-tritium gas). IECF device is an excellent apparatus because of its ability to generate fast neutrons with high-flux from a small source for many applications, such as medicine (e.g., boron neutron capture cancer therapy) [1-3], radiography or tomography of thick materials, space propulsion system [4], inspection system and explosive landmine detection [5], neutron activation analysis, mine and petroleum exploration, and security screening. Therefore neutron production rate optimization in this device will increase its efficiency for the above applications.

IEC researches have been performed since the 1950s decade. Recently, over the last 10 years, many researches and developments on IECF neutron source have been done $[2,4$, $6-8]$. Most of these developments are created by the usage of the different ion sources and enhancement of voltage and 
current $[2,4,6]$. IR-IECF is the prototype IEC device that is structured at the Atomic Energy Organization of Iran (AEOI) and the preliminary experiments on neutron yield were performed [7]. D-D neutrons have lower energy $(2.5 \mathrm{MeV})$ and yield compared with $\mathrm{D}-\mathrm{T}$ neutrons $(14 \mathrm{MeV})$ and therefore they can be moderated within a thinner moderation layer, and thermal neutron flux can be obtained with D-D neutrons. The mentioned benefit and other advantages of D-D neutrons caused that deuterium gas to be used in IECF device.

Reported results in previous works suggest that using the $\mathrm{D}$-T gas mixture and increasing the ion current via ion source would give a neutron rate of $7.3 \times 10^{12} \mathrm{n} / \mathrm{sec}$ at 1.2 mTorr, $75 \mathrm{kV}$, and $1.5 \mathrm{~A}$ ion current $[6,8,9]$. Researchers in Wisconsin University, in order to increase and optimize the neutron production rate in the UW IECF device, studied effect of cathode's size (diameter), geometry, and material composition on NPR [10]. Dietrich in MIT University investigated multigrid IEC device theoretically and experimentally for improving particle confinement [11]. In this study, for the first time, the ability of artificial neural network for modeling the IECF device in order to optimize the neutron production rate was investigated and the maximum neutron yield was obtained. In the recent work [12], dependence of neutron production rate (NPR) on cathode voltage and discharge current in IRIECF device for deuterium gas has been studied.

Objective of this work is to train an ANN based on the data presented in [12] to predict NPR at high voltages that cannot be achieved experimentally by the current facilities. The use of ANN is discussed in detail in the following sections to predict NPR for voltages up to $130 \mathrm{kV}$.

\section{Experimental Setup}

IR-IECF device consists of a spherical grounded anode, which serves as a chamber, and a grid cathode that are set concentrically as shown in Figure 1. This device consists of $13.5 \mathrm{~cm}$ diameter stainless steel cathode, $41 \mathrm{~cm}$ diameter anode with a $60 \mathrm{~cm}$ diameter, and $60 \mathrm{~cm}$ height vacuum chamber [7].

The IR-IECF facility accompanied with the cathode and the anode geometry is shown in Figure 2. In the experiments, facility is adjusted in a desired gas pressure and then by changing the cathode negative voltage glow plasma inside the cathode grid is produced. Produced ions are accelerated toward the cathode, and most of them penetrate the hollow cathode spherical wire net. In this situation, fusion reactions through beam-beam and beam-target collisions occurred. By increasing the cathode voltage and decreasing gas pressure, desired dense plasma is obtained.

In order to measure the neutron production rate (NPR), a calibrated neutron probe LB6411, which consists of a cylindrical ${ }^{3} \mathrm{He}$ proportional counter tube, was placed in $60 \mathrm{~cm}$ distance from cathode center of IR-IECF device [12]. The cathode voltage and discharge current have a significant impact on the NPR. The experiments had been carried out with the tunable $82 \mathrm{kV}$ DC high voltage. Maximum NPR was $1.46 \times 10^{7} \mathrm{n} / \mathrm{s}$ and had occurred when the voltage was $82 \mathrm{kV}$ and the discharge current was $48 \mathrm{~mA}$. In the next sections

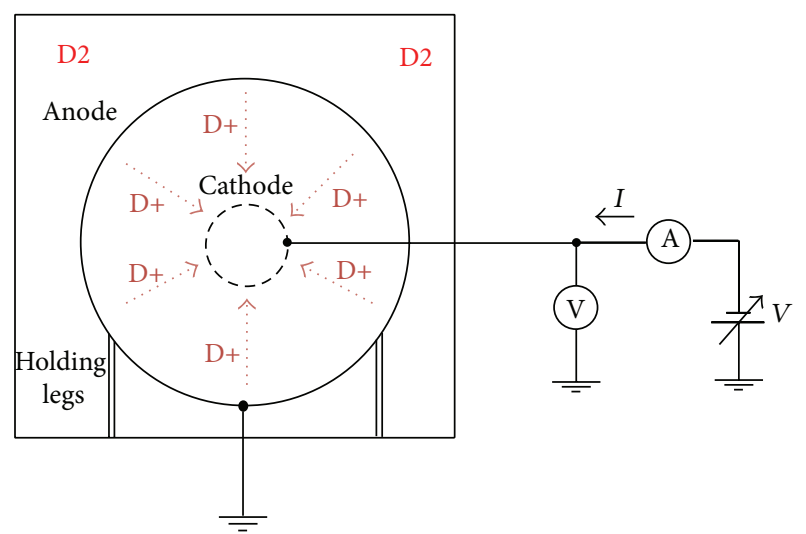

FIGURE 1: Schematic diagram of IR-IECF device and parameters used in this study.

using artificial neural network and these experimental data the NPR has been predicted for these ranges: current discharge $=40 \mathrm{~mA}-120 \mathrm{~mA}$ and voltage $=50 \mathrm{kV}-130 \mathrm{kV}$. The extrapolation beyond the range of experimental data is one of the most important applications of using ANNs to model the nonlinear unknown-response systems such as the studied IECF. This method has been used in many published works such as [13-15].

\section{Neural Network Computations}

Artificial neural networks (ANNs) are mathematical models inspired in the human brain. Ability of learning to use experimental data is the main characteristic of this technique. This model can predict behaviors and patterns from a finite set of experimental data, called the "training set" of the ANN [16]. In general, the structure of ANN typically is comprised of three layers including input, hidden, and output layers. First primary data is collected in input layer and then sent to different processing units (neuron), which constitute the hidden layer of the networks [17]. The structure of a neuron consists of two major terms of weight and activation function as shown in Figure 3.

Activation function receives an argument $n$ and generates an output $a$. The input of network for each neuron is sum of all input values in which each is multiplied by its weight and a bias term. The output value can be calculated by feeding the network input into the activation function of the neuron. Many activation functions are applied in ANN model such as triangular basis, pure line, soft max, log sigmoid, tan sigmoid, and hard limit. To achieve an acceptable prediction, the ANN is trained to minimize the error between ANN output and experimental data.

In this research, multilayer perceptron (MLP) neural networks were used. MLP is a feed-forward artificial neural network model that maps input data sets onto a set of appropriate outputs. What makes a multilayer perceptron a different tool to predict the nonlinear unknown-response systems is that each neuron uses a nonlinear activation function (such as tangent sigmoid), which was developed to model 


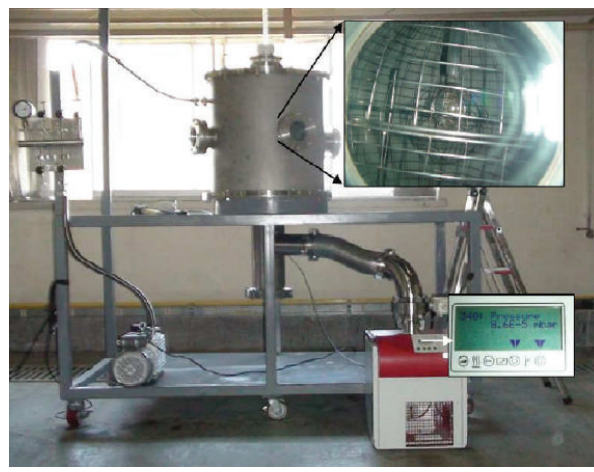

(a)

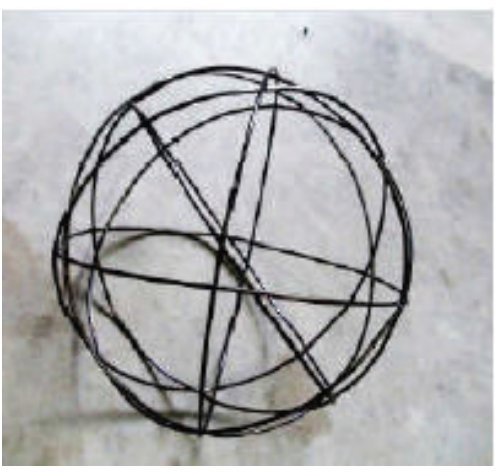

(b)

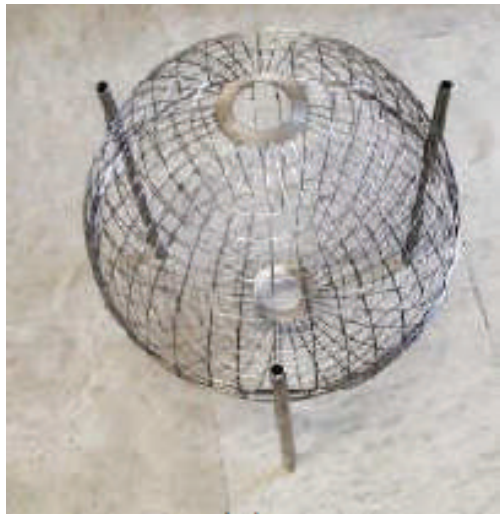

(c)

Figure 2: (a) IR-IECF device, (b) cathode ( $13.5 \mathrm{~cm}$ diameter), and (c) anode (41 cm diameter).

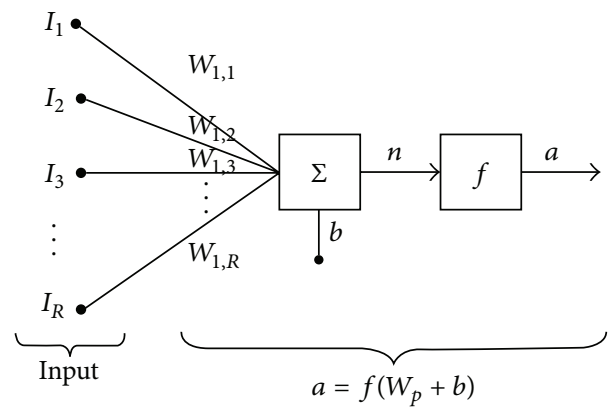

Figure 3: The neuron mathematical model.

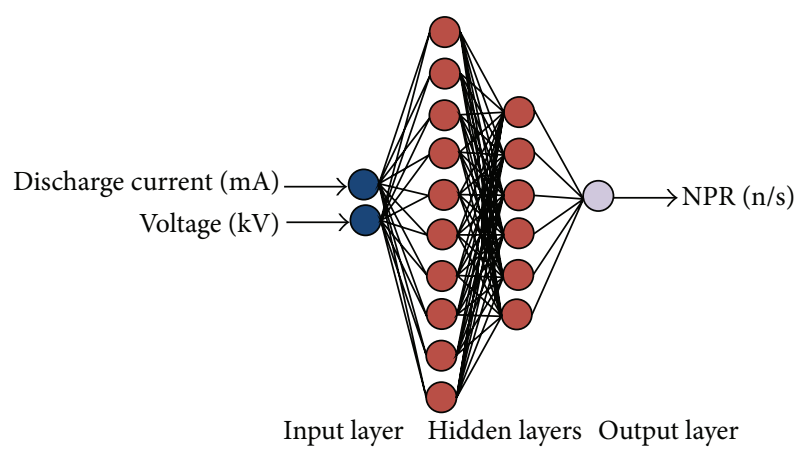

FIGURE 4: Architecture for the proposed MLP model.

the frequency of action potentials, or firing, of biological neurons in the brain $[18,19]$. The proposed MLP model is shown in Figure 4, where the inputs are discharge current $(\mathrm{mA})$ and voltage $(\mathrm{kV})$ and the output is NPR $(\mathrm{n} / \mathrm{s})$.

The input to the node $t$ in the first hidden layer is given by $[20,21]$

$$
\eta_{t}=\sum_{u=1}^{2}\left(X_{u} W_{u t}\right)+\theta_{t}, \quad t=1,2, \ldots, 10
$$

TABLE 1: Specification of the proposed ANN model.

\begin{tabular}{lc}
\hline Neural network & MLP \\
\hline Number of neurons in the input layer & 2 \\
Number of neurons in the first hidden layer & 10 \\
Number of neurons in the second hidden layer & 6 \\
Number of neurons in the output layer & 1 \\
Number of epochs & 1200 \\
Activation function & tansig \\
\hline
\end{tabular}

The output from the $t$ th neuron of the second hidden layer is given by

$$
O_{t}=f\left(\eta_{t}\right)
$$

where $X$ is the input variables, $\theta$ is the bias term, $W$ is the weighting factor, and $f$ is the activation function of the hidden layer. The output of the neuron in the output layer is given by

$$
Y=\sum_{u=1}^{6}\left(O_{u} W_{u}\right)+b
$$

Required data set for training the network is obtained by use of IR-IECF experimental data. The presented MLP network is trained by Levenberg-Marquardt (LM) algorithm [22]. In this method, first derivative and second derivative (Hessian) are used for network weight correction [23]. The numbers of samples for training and testing data are 36 (about 70\%) and 15 (about 30\%), respectively. In this study, for optimizing the ANN configuration many different structures with one hidden layer and two, three, and four hidden layers were tested by applying different number of neurons in each layer and different epochs. MATLAB 7.0.4 software was used for training the ANN model. Table 1 shows the specification of the proposed ANN model being used in this study. In this table, an epoch represents completion of processing on the entire training data set [23]. 


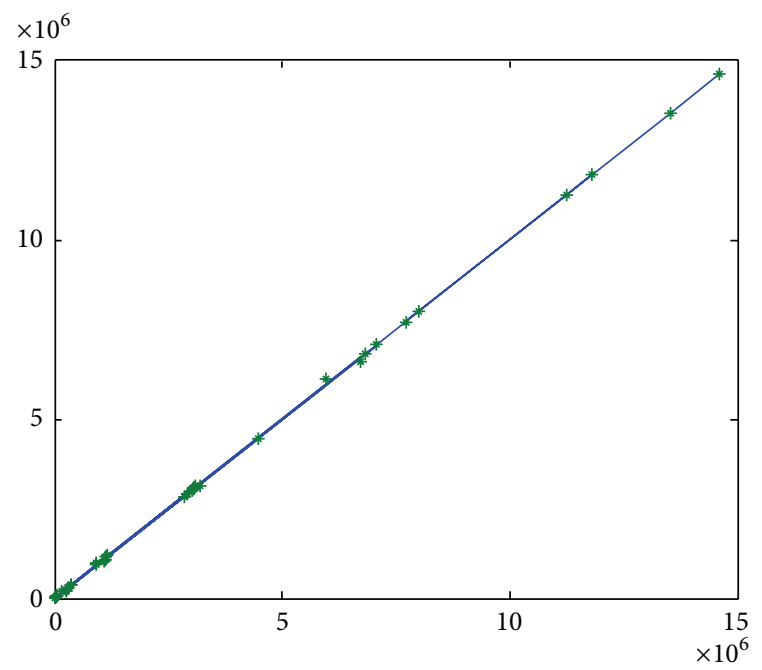

(a)

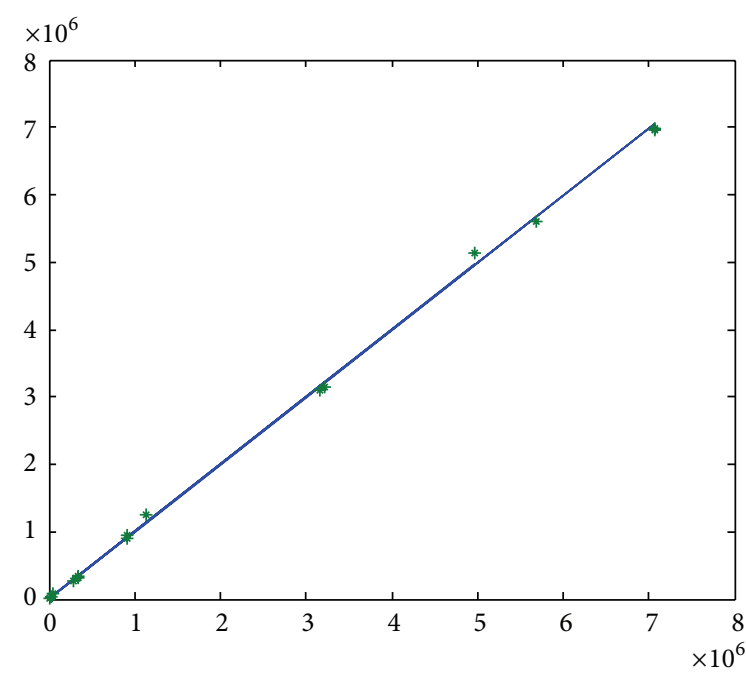

(b)

FIgURE 5: Comparison of experimental and predicted results for (a) training data and (b) testing data.
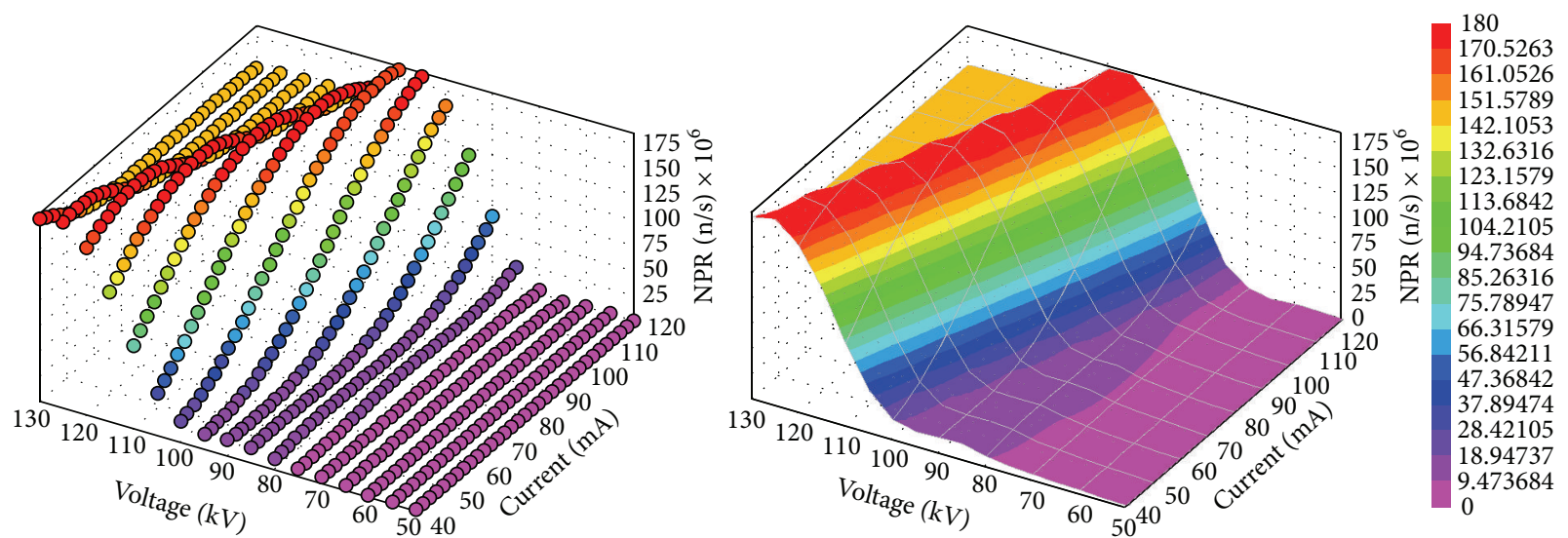

FIGURE 6: Prediction of NPR for current discharge $=40 \mathrm{~mA}-120 \mathrm{~mA}$ and voltage $=50 \mathrm{kV}-130 \mathrm{kV}$.

TABLE 2: Obtained errors for training and testing results of the proposed ANN model.

\begin{tabular}{lcc}
\hline Error & Train & Test \\
\hline MRE\% & 0.0072 & 0.6563 \\
RMSE & 0.0445 & 0.0719 \\
\hline
\end{tabular}

\section{Results and Dissection}

Table 2 shows the obtained errors for the proposed ANN model, where the mean relative error percentage (MRE\%) and the roots mean square error (RMSE) are calculated by

$$
\begin{aligned}
\operatorname{RMSE} & =\left[\frac{\sum_{m=1}^{N}\left(X_{m}(\operatorname{Exp})-X_{m}(\text { Pred })\right)^{2}}{N}\right]^{0.5}, \\
\text { MRE\% } & =100 \times \frac{1}{N} \sum_{m=1}^{N}\left|\frac{X_{m}(\text { Exp })-X_{m}(\text { Pred })}{X_{m}(\text { Exp })}\right|
\end{aligned}
$$

where $N$ is the number of data and " $X($ Exp)" and " $X$ (Pred)" stand for experimental and predicted (ANN) values, respectively.

Figure 5 shows the comparison of the experimental and predicted results using the proposed ANN model for training and testing data. The comparisons between experimental and predicted (ANN) results for training and testing data are listed in Tables 2 and 3, respectively.

For training results, variance and standard deviation of differences are obtained as $1.98 E-03$ and 0.0445 , respectively.

For testing result, the variance and standard deviation of differences are obtained as 0.005088 and 0.07134 , respectively. From Tables 3 and 4 and Figure 5, clearly the predicted produced neutrons by ANN model are close to the experimental results. These results show the applicability of ANN as an accurate and reliable model for the prediction of NPR in IECF device according to the discharge current and voltage. Figure 6 shows the prediction of NPR in the range of current discharge $=40 \mathrm{~mA}-120 \mathrm{~mA}$ and voltage $=50 \mathrm{kV}-130 \mathrm{kV}$. 
TABLE 3: The data that were used for training the network and predicted number of produced neutrons by ANN.

\begin{tabular}{|c|c|c|c|c|}
\hline Discharge current $(\mathrm{mA})$ & Voltage $(\mathrm{kV})$ & Produced neutron $\left(\times 10^{6}\right)$ & Predicted neutron $\left(\times 10^{6}\right)$ & Difference \\
\hline 20 & 30 & 0.055164 & 0.0552 & $-3.648 E-05$ \\
\hline 20 & 40 & 0.364079 & 0.3641 & $-2.08 E-05$ \\
\hline 20 & 60 & 2.846438 & 2.8464 & $3.8 E-05$ \\
\hline 20 & 70 & 4.468245 & 4.4682 & $4.5 E-05$ \\
\hline 30 & 40 & 0.358563 & 0.3667 & -0.0081371 \\
\hline 30 & 50 & 1.152918 & 1.2269 & -0.073982 \\
\hline 30 & 60 & 3.033994 & 3.0024 & 0.031594 \\
\hline 40 & 30 & 0.052405 & 0.0584 & -0.0059947 \\
\hline 40 & 50 & 1.130852 & 1.1437 & -0.012848 \\
\hline 40 & 60 & 3.072608 & 3.0582 & 0.014408 \\
\hline 50 & 30 & 0.042476 & 0.0469 & -0.0044241 \\
\hline 50 & 50 & 1.125336 & 1.0738 & 0.051536 \\
\hline 50 & 70 & 5.95766 & 6.1068 & -0.14914 \\
\hline 60 & 40 & 0.292367 & 0.2832 & 0.0091667 \\
\hline 60 & 50 & 1.10327 & 1.0192 & 0.08407 \\
\hline 60 & 70 & 6.729949 & 6.5857 & 0.144249 \\
\hline 70 & 30 & 0.027582 & 0.029 & -0.0014182 \\
\hline 70 & 50 & 0.926747 & 0.9802 & -0.0534529 \\
\hline 70 & 60 & 3.199484 & 3.155 & 0.044484 \\
\hline 80 & 30 & 0.017652 & 0.0214 & -0.0037477 \\
\hline 80 & 40 & 0.248236 & 0.2421 & 0.0061358 \\
\hline 80 & 60 & 3.089157 & 3.1426 & -0.053443 \\
\hline 80 & 70 & 7.060931 & 7.0959 & -0.034969 \\
\hline 90 & 40 & 0.24272 & 0.2172 & 0.0255195 \\
\hline 90 & 50 & 0.910198 & 0.9312 & -0.0210019 \\
\hline 90 & 60 & 3.056059 & 3.0759 & -0.019841 \\
\hline 100 & 30 & 0.012136 & 0.0121 & $3.597 E-05$ \\
\hline 100 & 40 & 0.171007 & 0.1823 & -0.0112931 \\
\hline 100 & 60 & 2.923667 & 2.8965 & 0.027167 \\
\hline 100 & 70 & 6.840276 & 6.8374 & 0.002876 \\
\hline 50 & 75 & 7.99871 & 7.988 & 0.01071 \\
\hline 96 & 77 & 11.80499 & 11.8062 & -0.001207 \\
\hline 55 & 77 & 11.25336 & 11.2547 & -0.001342 \\
\hline 26 & 81 & 7.722893 & 7.7191 & 0.003793 \\
\hline 75 & 82 & 13.51506 & 13.513 & 0.002062 \\
\hline 48 & 82 & 14.61833 & 14.6195 & -0.001167 \\
\hline
\end{tabular}

In this range, maximum number of produced neutrons happened in $I=45 \mathrm{~mA}$ and $V=125 \mathrm{kV}$. In this situation, IECF can produce $1.855 \times 10^{8}$ neutrons per second. In another optimum point where $I=72.5 \mathrm{~mA}$ and $V=115 \mathrm{kV}$, the IECF can produce $1.854 \times 10^{8}$ neutrons per second. These results show significant NPR increase in the IECF device according to increasing voltage. $52 \%$ increment in maximum voltage of power supply ( $82 \mathrm{kV}$ to $125 \mathrm{kV}$ ) was found and, in optimum operational discharge current (in this case $I=45 \mathrm{~mA}$ ), the neutron produced rate can be increased to $1270 \% \mathrm{NPR}(1.46 \times$ $10^{7} \mathrm{n} / \mathrm{s}$ to $1.855 \times 10^{8} \mathrm{n} / \mathrm{s}$ ).

\section{Conclusion}

In this paper, the development of an ANN model for prediction of the neutron yield in IECF devices is presented. The comparison between experimental and predicted results by ANN model shows that there is a good agreement between them with mean relative error (MRE) less than 0.66\%. Therefore, the MLP network can be used as an efficient tool to predict the NPR in the IECF device. For developing the model, the input parameters are voltage $(\mathrm{kV})$ and discharge current $(\mathrm{mA})$ and the output is neutron yield $(\mathrm{n} / \mathrm{s})$. Results 
TABLE 4: The data that were used for testing the network and predicted number of produced neutrons by ANN.

\begin{tabular}{|c|c|c|c|c|}
\hline Discharge current $(\mathrm{mA})$ & Voltage $(\mathrm{kV})$ & Produced neutron $\left(\times 10^{6}\right)$ & Predicted neutron $\left(\times 10^{6}\right)$ & Difference \\
\hline 20 & 50 & 1.130852 & 1.2495 & -0.11865 \\
\hline 30 & 30 & 0.052957 & 0.0723 & -0.01934 \\
\hline 30 & 70 & 4.964717 & 5.131 & -0.16628 \\
\hline 40 & 40 & 0.34753 & 0.3329 & 0.01463 \\
\hline 40 & 70 & 5.681843 & 5.6062 & 0.075643 \\
\hline 50 & 60 & 3.166386 & 3.1047 & 0.061686 \\
\hline 50 & 40 & 0.342014 & 0.3058 & 0.036214 \\
\hline 60 & 30 & 0.035856 & 0.0373 & -0.00144 \\
\hline 60 & 60 & 3.210517 & 3.1387 & 0.071817 \\
\hline 70 & 40 & 0.28685 & 0.2629 & 0.02395 \\
\hline 70 & 70 & 7.060931 & 6.955 & 0.105931 \\
\hline 80 & 50 & 0.915714 & 0.9536 & -0.03789 \\
\hline 90 & 30 & 0.014894 & 0.0133 & 0.001594 \\
\hline 90 & 70 & 7.060931 & 6.9842 & 0.076731 \\
\hline 100 & 50 & 0.904682 & 0.8952 & 0.009482 \\
\hline
\end{tabular}

show that in optimum discharge current when maximum voltage of power supply increases 1.5 times, NPR can increase more than 12 times.

\section{Conflict of Interests}

The authors declare that there is no conflict of interests regarding the publication of this paper.

\section{References}

[1] G. L. Kulcinski, "Non-electric applications of fusion energyan important precursor to commercial electric power," Fusion Technology, vol. 34, no. 3, pp. 477-483, 1998.

[2] R. P. Ashley and the UW IEC Team, "Experimental progress in 2003 of the UW IEC facility," in Proceedings of the 6th U.S.-Japan IEC Workshop, Tokyo, Japan, October 2003.

[3] R. P. Ashley, G. L. Kulcinski, J. F. Santarius, S. K. Murali, G. Piefer, and R. Radel, "Steady-state D3He proton production in an IEC fusion device," Fusion Technology, vol. 39, no. 2, pp. 546$551,2001$.

[4] C. C. Dobson and I. Hrbud, "Electron density and two-channel neutron emission measurements in steady-state spherical inertial-electrostatically confined plasmas, with review of the one-dimensional kinetic model," Journal of Applied Physics, vol. 96, no. 1, pp. 94-108, 2004.

[5] K. Yoshikawa, K. Masuda, T. Takamatsu et al., "Research and development on a compact discharge-driven D-D fusion neutron source for explosive detection," in Proceedings of the 2nd Joint International Conference on Sustainable Energy and Environment (SEE '06), Bangkok, Thailand, November 2006.

[6] T. Takamatsu, K. Masuda, T. Kyunai, H. Toku, and K. Yoshikawa, "Inertial electrostatic confinement fusion device with an ion source using a magnetron discharge," Nuclear Fusion, vol. 46, no. 1, pp. 142-148, 2006.

[7] V. Damideh, A. Sadighzadeh, A. Koohi et al., "Experimental study of the Iranian inertial electrostatic confinement fusion device as a continuous neutron generator," Journal of Fusion Energy, vol. 31, no. 2, pp. 109-111, 2012.
[8] G. H. Miley, Y. Yang, J. Webber, Y. Shaban, and H. Momota, "RF ion source-driven IEC design and operation," Fusion Science and Technology, vol. 47, no. 4, pp. 1233-1237, 2005.

[9] G. H. Miley, Y. Shaban, and Y. Yang, "RF ion gun injector in support of fusion ship II research and development," AIP Conference Proceedings, vol. 699, no. 1, p. 406, 2004.

[10] A. L. Wehmeyer, R. F. Radel, and G. L. Kulcinski, “Optimizing neutron production rates from DD fusion in an inertial electrostatic confinement device," in Proceedings of the 6th ANS Topical Meeting on Fusion Energy, Madison, Wis, USA, September 2004.

[11] C. C. Dietrich, Improving particle confinement in inertial electrostatic fusion for spacecraft power and propulsion [Ph.D. thesis], MIT University, 2007.

[12] E. H. Ebrahimi, R. Amrollahi, A. Sadighzadeh et al., "The influence of cathode voltage and discharge current on neutron production rate of inertial electrostatic confinement fusion (IRIECF)," Journal of Fusion Energy, vol. 32, no. 1, pp. 62-65, 2013.

[13] H. K. Cigizoglu, "Estimation, forecasting and extrapolation of river flows by artificial neural networks," Hydrological Sciences Journal, vol. 48, no. 3, pp. 349-362, 2003.

[14] Z. Liu and J. Liu, "Seismic-controlled nonlinear extrapolation of well parameters using neural networks," Geophysics, vol. 63, no. 6, pp. 2035-2041, 1998.

[15] Ö. Kiși, "Streamflow forecasting using different artificial neural network algorithms," Journal of Hydrologic Engineering, vol. 12, no. 5, pp. 532-539, 2007.

[16] C. M. Salgado, L. E. B. Brandão, R. Schirru, C. M. N. A. Pereira, A. X. da Silva, and R. Ramos, "Prediction of volume fractions in three-phase flows using nuclear technique and artificial neural network," Applied Radiation and Isotopes, vol. 67, no. 10, pp. 1812-1818, 2009.

[17] F. Rahimi-Ajdadi and Y. Abbaspour-Gilandeh, "Artificial Neural Network and stepwise multiple range regression methods for prediction of tractor fuel consumption," Measurement, vol. 44, no. 10, pp. 2104-2111, 2011. 
[18] F. J. de Cos Juez, M. A. Suárez-Suárez, F. Sánchez Lasheras, and A. Murcia-Mazón, "Application of neural networks to the study of the influence of diet and lifestyle on the value of bone mineral density in post-menopausal women," Mathematical and Computer Modelling, vol. 54, no. 7-8, pp. 1665-1670, 2011.

[19] A. Ebrahimzadeh and A. Khazaee, "Detection of premature ventricular contractions using MLP neural networks: a comparative study," Measurement, vol. 43, no. 1, pp. 103-112, 2010.

[20] A. R. Gallant and H. White, "On learning the derivatives of an unknown mapping with multilayer feedforward networks," Neural Networks, vol. 5, pp. 129-138, 1992.

[21] J. G. Taylor, Neural Networks and Their Applications, John Wiley \& Sons, West Sussex, UK, 1996.

[22] M. T. Hagan and M. B. Menhaj, "Training feedforward networks with the Marquardt algorithm," IEEE Transactions on Neural Networks, vol. 5, no. 6, pp. 989-993, 1994.

[23] B.-H. Juang and S. Katagiri, "Discriminative learning for minimum error classification," IEEE Transactions on Signal Processing, vol. 40, no. 12, pp. 3043-3054, 1992. 

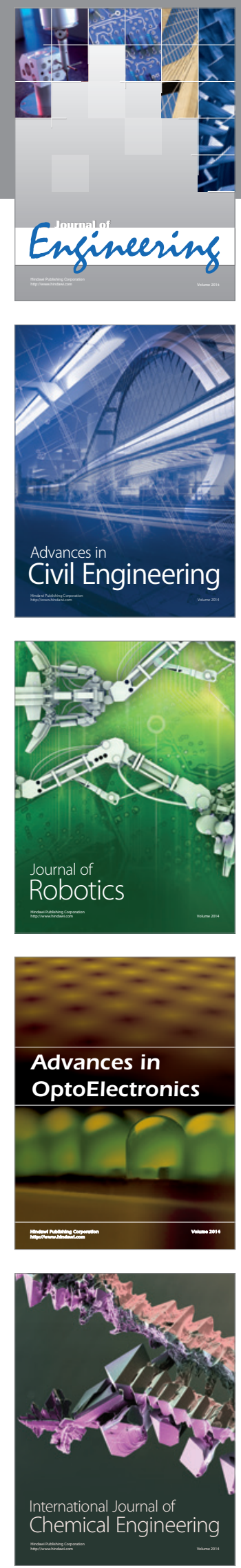

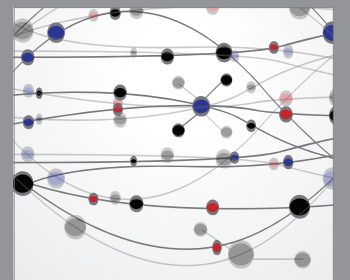

The Scientific World Journal
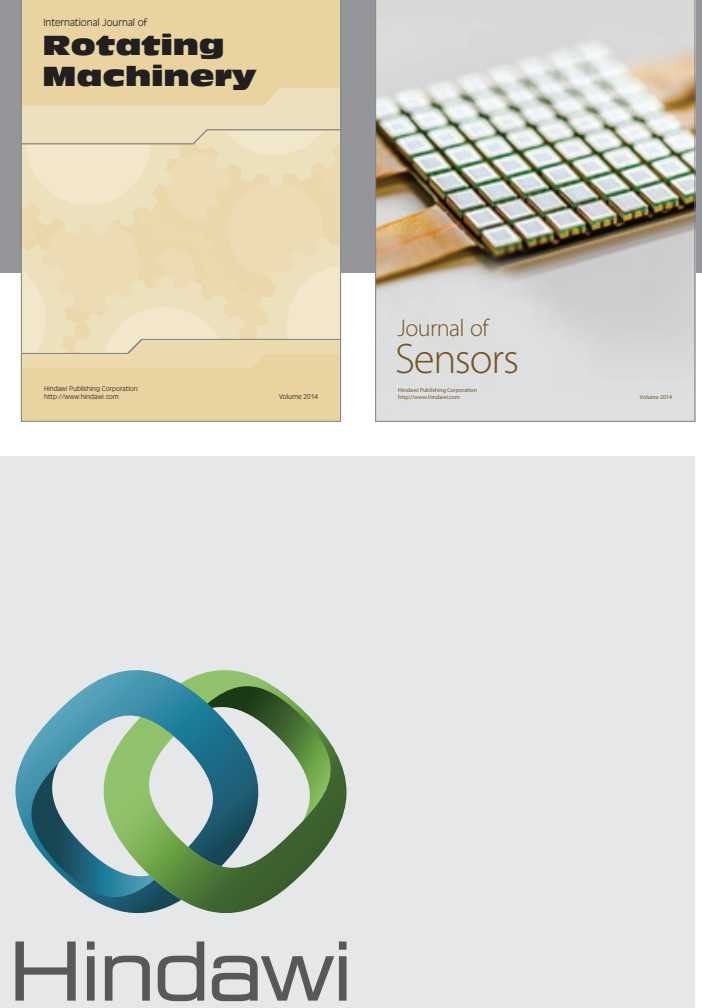

Submit your manuscripts at http://www.hindawi.com
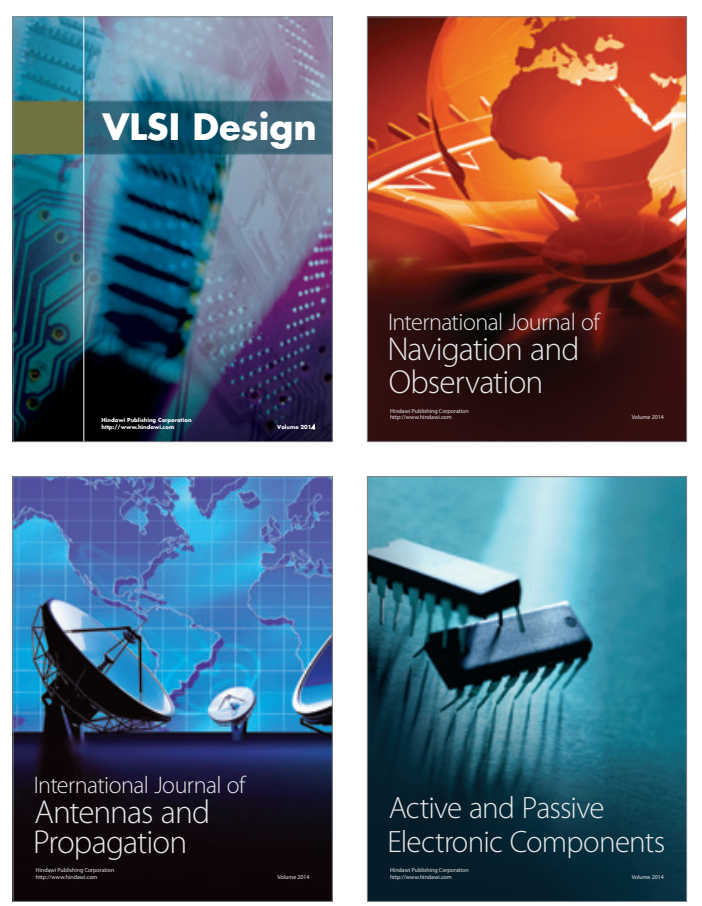
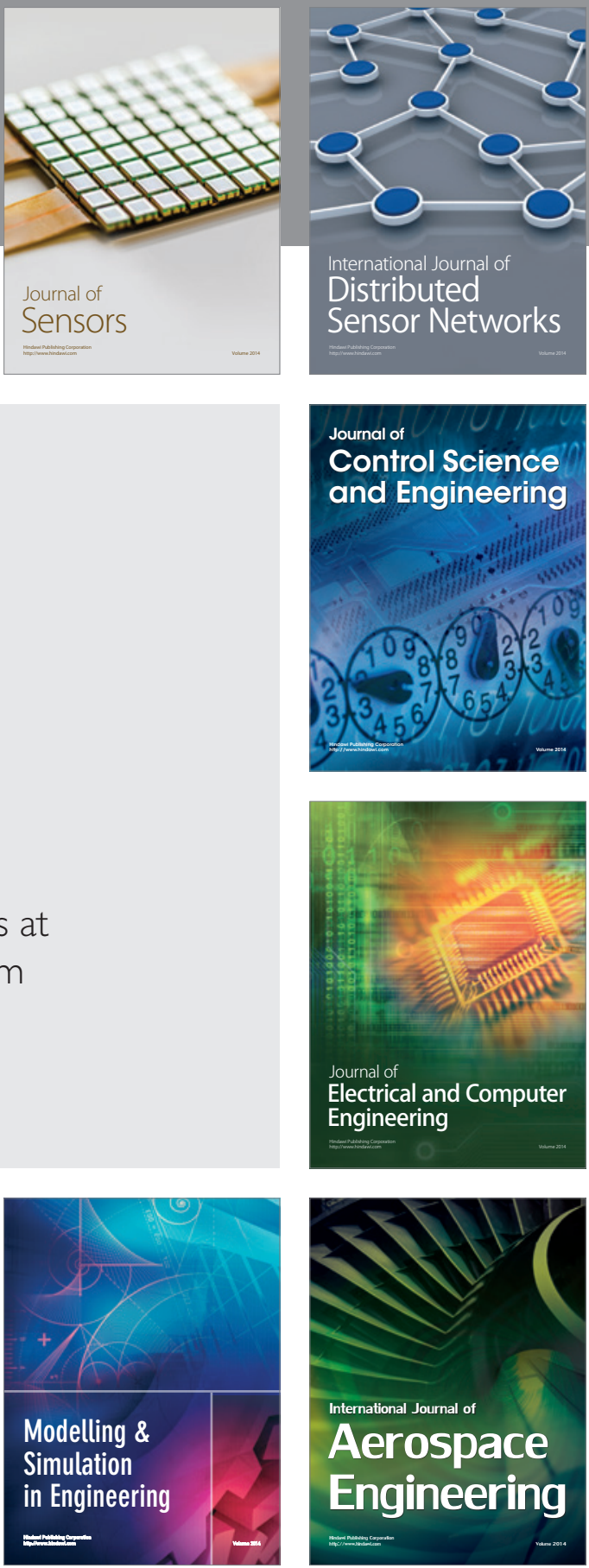

Journal of

Control Science

and Engineering
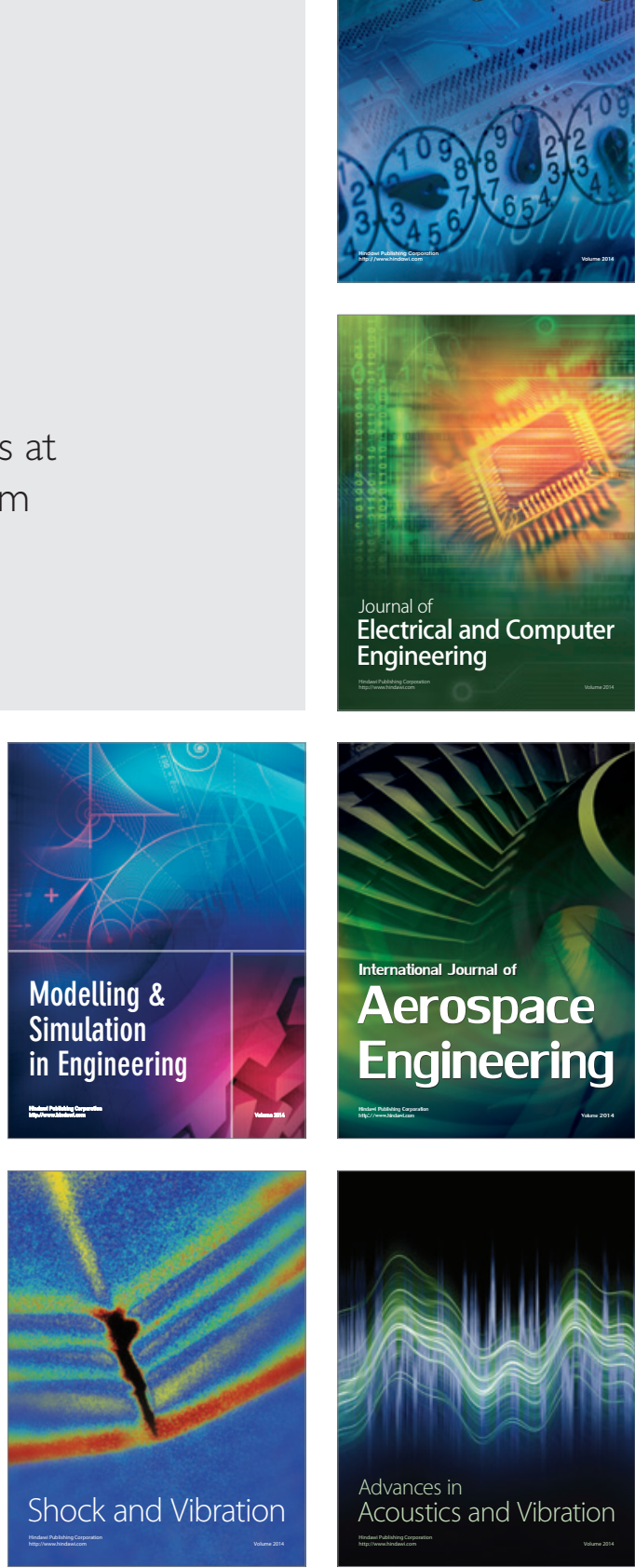